\title{
Correction to: Chronic Aichi Virus Infection in a Patient with X-Linked Agammaglobulinemia
}

\author{
Giorgia Bucciol $^{1,2}$ - Leen Moens ${ }^{1} \cdot$ Kathryn Payne $^{3}$ - Elke Wollants ${ }^{4}$ - Djalila Mekahli ${ }^{2,5}$ - Elena Levtchenko ${ }^{2,5}$. \\ François Vermeulen $^{2}$. Thomas Tousseyn ${ }^{6}$ - Paul Gray ${ }^{7,8}$. Cindy S. Ma ${ }^{3,9,10}$ - Stuart G. Tangye ${ }^{3,11,10}$. \\ Marc Van Ranst ${ }^{4,12} \cdot$ Julianne R. Brown ${ }^{9} \cdot$ Judy Breuer ${ }^{9,13} \cdot$ Isabelle Meyts ${ }^{1,2}$ (ID
}

Published online: 14 November 2018

(C) Springer Science+Business Media, LLC, part of Springer Nature 2018

\section{Correction to: Journal of Clinical Immunology https://doi.org/10.1007/s10875-018-0558-z}

The original version of this article unfortunately did not display the appropriate captions in the figure. The correct version is displayed below.

The online version of the original article can be found at https://doi.org/ 10.1007/s10875-018-0558-Z

Isabelle Meyts

Isabelle.Meyts@uzleuven.be

1 Laboratory of inborn errors of immunity, Department of Immunology and Microbiology, KU Leuven, Leuven, Belgium

2 Department of Pediatrics, University Hospitals Leuven, Herestraat, 49, 3000 Leuven, Belgium

3 Immunology Division, Garvan Institute of Medical Research, Darlinghurst, NSW, Australia

4 Laboratory of Clinical and Epidemiological Virology (Rega Institute), KU Leuven, Leuven, Belgium

5 Department of Development and Regeneration, KU Leuven, Leuven, Belgium

6 Department of Imaging \& Pathology, KU Leuven and University Hospitals Leuven, Leuven, Belgium
7 School of Women's and Children's Health, Faculty of Medicine, University of New South Wales, Sydney, NSW, Australia

8 Department of Immunology and Infectious Disease, Sydney Children's Hospital, Sydney, NSW, Australia

9 St Vincent's Clinical School, UNSW Sydney Faculty of Medicine, Sydney, NSW, Australia

10 CIRCA (Clincal Immunogenomics Research Consortia Australia), Sydney, NSW, Australia

11 Department of Microbiology and Immunology, KU Leuven, Leuven, Belgium

12 Department of Microbiology, Virology and Infection Prevention and Control, Great Ormond Street Hospital for Children NHS Foundation Trust, London, UK

13 Division of Infection and Immunity, University College London, London, UK 
A

T cells

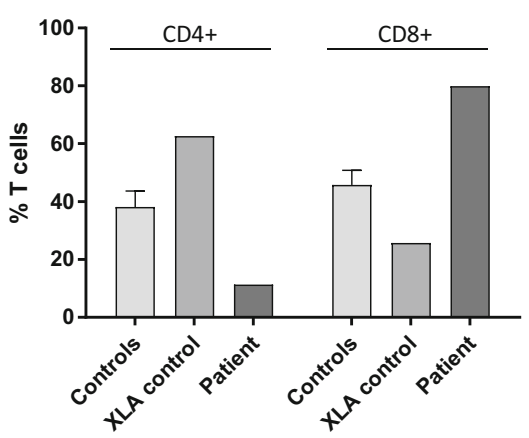

D
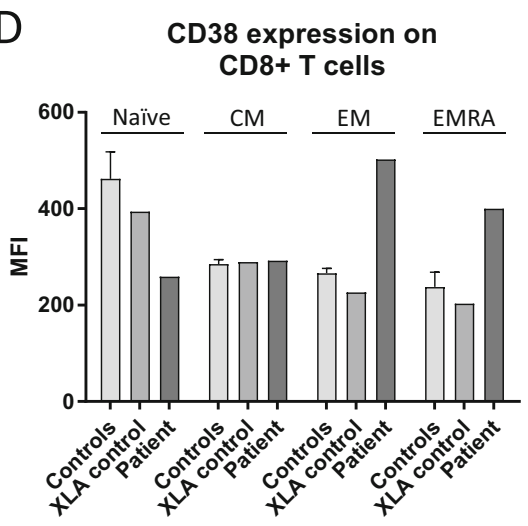

G CD95 expression on CD8+ T cells

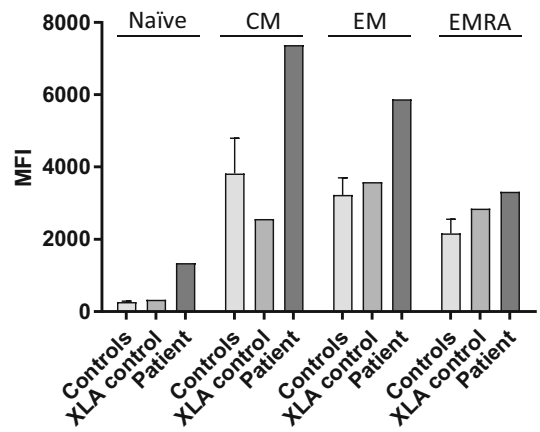

B

CD4+ T cell populations

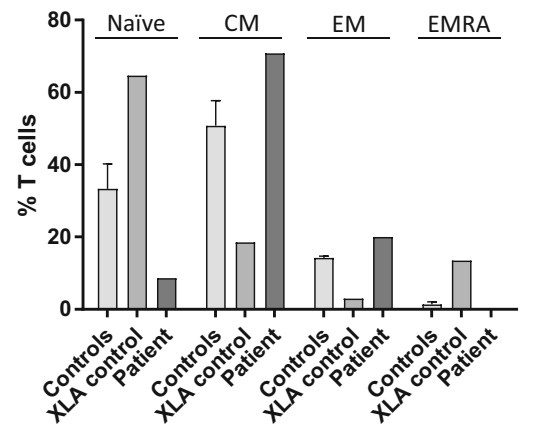

$E$
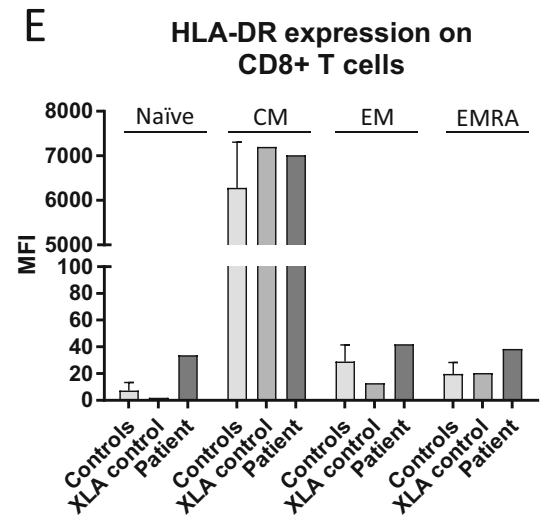

$\mathrm{H}$
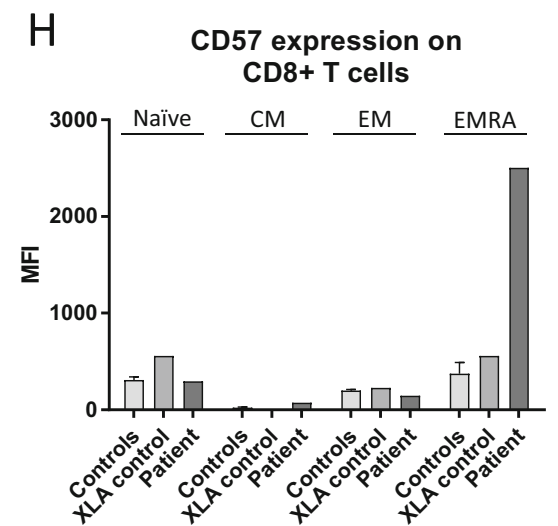

C

CD8+ T cell populations

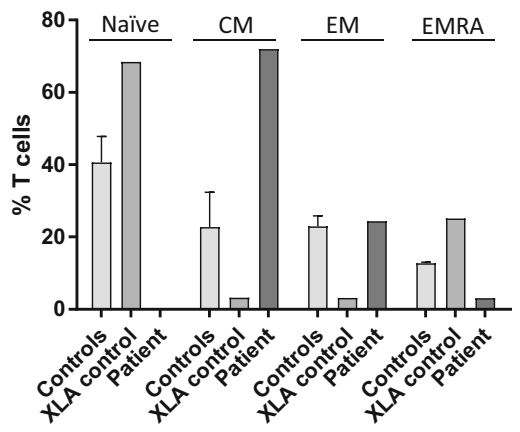

$\mathrm{F}$

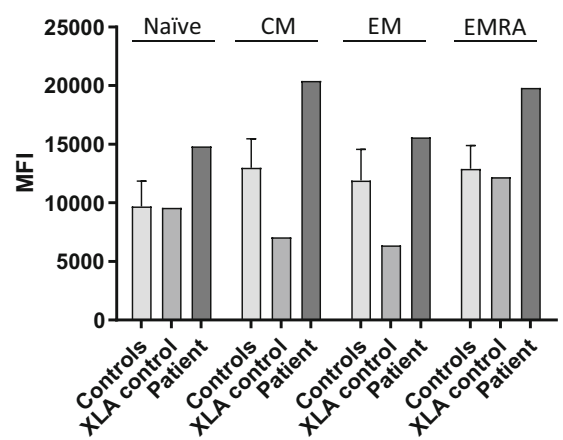

I

\section{CX3CR1 expression on} CD8+ T cells

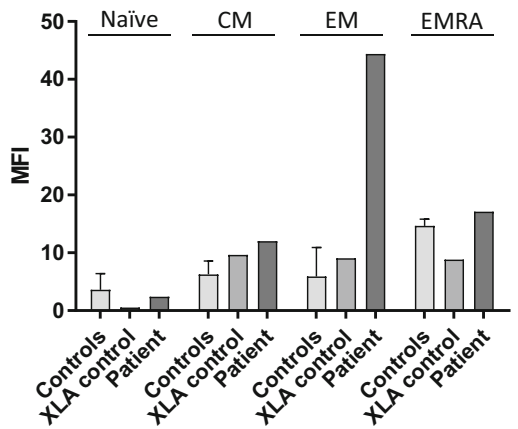

$\square$ Controls $\quad \square$ XLA control $\quad \square$ Patient

Fig. 1 CD8+ T cells in the proband XLA patient are skewed towards a memory phenotype with evidence of exhaustion and/or senescence. PBMCs from healthy controls, an XLA control and our XLA patient were labeled with mAbs against CD4, CD8, CD45RA, CCR7, CD38, HLADR, HLA-ABC, CD95, CD57, and CX3CR1. Proportions of (A) CD4 ${ }^{+}$ and $\mathrm{CD} 8^{+} \mathrm{T}$ cell populations, as well as subsets of naïve $\left(\mathrm{CD} 45 \mathrm{RA}^{+} \mathrm{CCR} 7^{+}\right)$, central memory $\left(\mathrm{CM}, \mathrm{CD} 45 \mathrm{RA}^{-} \mathrm{CCR} 7^{+}\right)$, effector memory (EM, CD45RA ${ }^{-} \mathrm{CCR}^{-}$), and terminally differentiated effector memory cells expressing CD45RA (EMRA, CD45RA ${ }^{+} \mathrm{CCR} 7^{-}$), (B) $\mathrm{CD}^{+}$, and $(\mathrm{C}) \mathrm{CD}^{+} \mathrm{T}$ cells were delineated. Differential expression of the activation markers CD38 (D), HLA-DR (E), and HLA-ABC (F), as well as the exhaustion/senescence markers CD95 (G), CD57 (H) and CX3CR1 (I) on naïve, $\mathrm{T}_{\mathrm{CM}}, \mathrm{T}_{\mathrm{EM}}$ and $\mathrm{T}_{\mathrm{EMRA}} \mathrm{CD}^{+} \mathrm{T}$ cells were determined. Values represent the geometric MFI 